Int. J. Dev. Biol. 56: 301-309

doi: $10.1387 / \mathrm{ijdb} .113327 \mathrm{p}$

\title{
Sarcosin (Krp1) in skeletal muscle differentiation: gene expression profiling and knockdown experiments
}

\author{
LEONIE DU PUY',\#, ABDELAZIZ BEQQALI,"\#\#, HELENA TA VAN TOL', JANTINE MONSHOUWER-KLOOTS², \\ ROBERT PASSIER ${ }^{2}$, HENK P. HAAGSMAN ${ }^{3}$ and BERNARD A.J. ROELEN ${ }^{*}, 1,4$ \begin{abstract}
of Anatomy \& Embryology, Leiden University Medical Center, Leiden, ${ }^{3}$ Department of Infectious Diseases and Immunology, Faculty of Veterinary Medicine, Utrecht University, Utrecht and ${ }^{4}$ Department of Equine Sciences,
\end{abstract} \\ ${ }^{1}$ Department of Farm Animal Health, Faculty of Veterinary Medicine, Utrecht University, Utrecht, ${ }^{2}$ Department \\ Faculty of Veterinary Medicine, Utrecht University, Utrecht, the Netherlands.
}

\begin{abstract}
SARCOSIN, also named Krp1, has been identified as a protein exclusively expressed in striated muscle tissue. Here we report on the role of SARCOSIN in skeletal muscle development and differentiation. We demonstrate, by means of whole-mount in situ hybridization, that Sarcosin mRNA is expressed in the myotome part of the mature somites in mouse embryos from embryonic day 9.5 onwards. Sarcosin is not expressed in the developing heart at these embryonic stages, and in adult tissues the mRNA expression levels are five times lower in the heart than in skeletal muscle. SARCOSIN protein partially co-localizes with the M-band protein myomesin and between and below laterally fusing myofibrils in adult skeletal muscle tissue. RNA interference mediated knock-down of SARCOSIN in the C2C12 myoblast cell line appeared to be stimulatory in the early phase of differentiation, but inhibitory at a later phase of differentiation.
\end{abstract}

KEY WORDS: mouse, skeletal muscle, sarcosin, differentiation, RNAi

\section{Introduction}

SARCOSIN, also named kelch related protein 1 (Krp1) was originally identified and described to be exclusively expressed in sarcomeric muscle (Taylor et al., 1998). Northern hybridization experiments revealed high expression in adult human skeletal and heart muscle, with lower levels of expression in prostate muscle. The levels in skeletal muscle were found to be about 15 fold greater than the levels in cardiac muscle. No hybridization was detected in muscle samples from uterus, colon, intestine, bladder and stomach (Taylor et al., 1998).

In skeletal muscle differentiation, important processes take place such as withdrawal from the cell cycle, fusion, de novo myofibrillogenesis and myotube formation that do not occur in primary cardiomyocyte cultures but the function of SARCOSIN in these processes is relatively unknown.

Skeletal muscle originates from the mesoderm. During embryonic development, paraxial mesoderm is first present on either side of the neural tube and notochord after which it will undergo segmentation to form somites. The somites are further specified into ventral sclerotome, which gives rise to the axial skeleton, and dorsal dermomytome responsible for the formation of dermal precursors and trunk, limb and several head muscles (Buckingham, 2001).

Because of relatively high expression levels in skeletal muscle, SARCOSIN is thought to be important in muscle physiology but little is known about its expression during embryonic development. Several indications for SARCOSIN's functions are presented by its structure. The amino acid sequence encodes five Kelch repeats at its carboxyl terminus and a BTB/POZ domain at the amino terminus (Spence et al., 2000; Taylor et al., 1998). Kelch repeats form a $\beta$-propeller which is important for interactions with proteins of the cytoskeleton (Gray et al., 2009) and proteins containing Kelch repeats indeed have diverse functions in cell morphology and organization (Adams et al., 2000).

Several proteins have been reported as binding partners for SARCOSIN, all alluding to a role in cell structure and in particular in myofibril function. One of such binding partners of SARCOSIN is

Abbreviations used in this paper: ISH, in situ hybridisation; N-RAP, nebulin-related anchoring protein; siRNA, small interfering RNA.

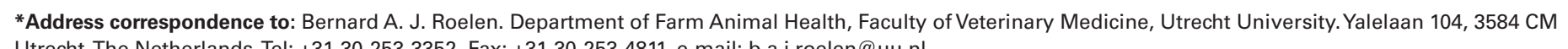
Utrecht, The Netherlands. Tel: +31-30-253-3352. Fax:+31-30-253-4811. e-mail: b.a.j.roelen@uu.nl

Present addresses: "Kinesis-Pharma, Breda, the Netherlands; \#\#Heart Failure Research Center, Academic Medical Center, Amsterdam, the Netherlands 
NEBULIN (Spence et al., 2006), an actin-binding protein thought to function as a 'ruler' to regulate the precise lengths of the actin (thin) filaments in skeletal muscle (Witt et al., 2006). Also, NEBULIN can interact with the intermediate filament DESMIN, possibly laterally linking Z-lines and integrating myofibrils with the cell membrane (Bang et al., 2002). SARCOSIN further can be a binding partner of Nebulin-related anchoring protein (N-RAP), an actin-binding protein expressed in skeletal and cardiac muscle tissues (Lu et al., 2003). N-RAP is expressed in the regions where actin-bundles link myofibrils to the cell membrane (Herrera et al., 2000; Zhang et al., 2001). In embryonic cardiac cells N-RAP is associated with premyofibril structures and newly formed mature myofibrils (Lu etal., 2005), while in skeletal muscle it has been identified in developing myofibrillar structures, but not in mature myofibrils (Lu etal., 2008).

Immunofluorescence staining in cultured chick cardiomyocytes showed localization of SARCOSIN between narrow myofibrils that appear to be fusing laterally (Lu et al., 2003). SARCOSIN knock-down by RNAi in cultured mouse embryonic cardiomyocytes resulted in an $\alpha$-ACTININ staining pattern that is characteristic of newly forming myofibrils suggesting that SARCOSIN affects the assembly and or maintenance of myofibril structure (Greenberg et al., 2008). Very recently, it was described that SARCOSIN expression is upregulated in $\mathrm{C} 2$ cells differentiating to myoblastst. Intriguingly, both knockdown and overexpression of SARCOSIN in these cells inhibited myoblast differentiation (Paxton et al., 2011).

Besides its expression in striated muscle, SARCOSIN was also up-regulated in v-Fos transformed rat fibroblasts which became invasive and underwent extensive cytoskeletal reorganizations forming long pseudopodia. SARCOSIN localized at the tip of these pseudopodia, and while over-expression of SARCOSIN resulted in elongated pseudopodia, small interfering RNA (siRNA) mediated down-regulation caused shortening of these structures. The function of SARCOSIN in pseudopodia elongation is dependent on the binding of SARCOSIN to LIM and SH3 protein (LASP-1) (Spence et al., 2000; Spence et al., 2006). LASP-1 is expressed in almost all adult mouse tissues and over-expressed in human breast cancers (Schreiber et al., 1998a; Tomasetto et al., 1995). Interestingly, LASP-1 also contains two nebulin repeats, binds to non-muscle F-actin in vitro and is localized to focal adhesions and pseudopodia (Chew et al., 2002; Lin et al., 2004; Schreiber et al., 1998b). A specific role for LASP-1 in muscle has not been described.

In this study the expression of Sarcosin mRNA during mouse embryonic development was visualized by whole-mount in situ hybridization (ISH) and SARCOSIN protein expression in adult skeletal muscle by immunofluorescence. SARCOSIN's role in the differentiation of skeletal muscle cells was studied by siRNA mediated down-regulation of SARCOSIN in the C2C12 myoblast cell line, which is an established cell line to study myogenesis.

\section{Results}

\section{Sarcosin mRNA is expressed in the somites during mouse embryonic development}

In order to investigate the expression of Sarcosinduring development we performed whole-mount ISH on mouse embryos. Sarcosin mRNA was first observed in embryonic day (E)9.5 embryos, and expression was exclusively observed in the oldest somites (Fig .1A, B). At E10.5 SarcosinmRNAwas expressed in all somites (Fig. 1C). In contrast to what we expected, Sarcosin was not expressed in the embryonic heart during the period examined (E8-E10.5). Sagittal sections of whole-mount ISH of E9.5 embryos demonstrated Sarcosin expression in what is most likely the myotome part of the somites(Fig. 1D). In transverse sections, the Sarcosinlabel was too weak to determine its localization with certainty (data no shown). Whole-mount ISH was performed on embryos from E8.5-E10.5. At those stages, the only Sarcosin expression observed was in the somites, no expression was observed in other skeletal muscle tissue such as cranial or pharyngeal muscle. Quantitative real-time PCR analysis demonstrated expression in mouse adult heart and skeletal muscle, with expression levels of Sarcosin mRNA in the heart being approximately five times lower compared to skeletal muscle expression (Fig. 1E).

\section{SARCOSIN is localized between laterally fusing myofibrils in adult skeletal muscle}

The localization of SARCOSIN protein in adult skeletal muscle was determined using immunofluorescence. For orientation within the muscle, Z-discs were visualized by $\alpha$-ACTININ staining (Fig. 2 A-D) and M-bands by MYOMESIN staining (Fig. 2 E-H). In adult skeletal muscle tissue an overall staining of SARCOSIN was observed, and SARCOSIN partially co-localized with the M-band protein MYOMESIN (Fig. 2 F-G) and was localized between (Fig 2B,D) and below laterally fusing myofibrils (Fig. 2 C,D). Stainings with isotype control antibodies were negative (data not shown).

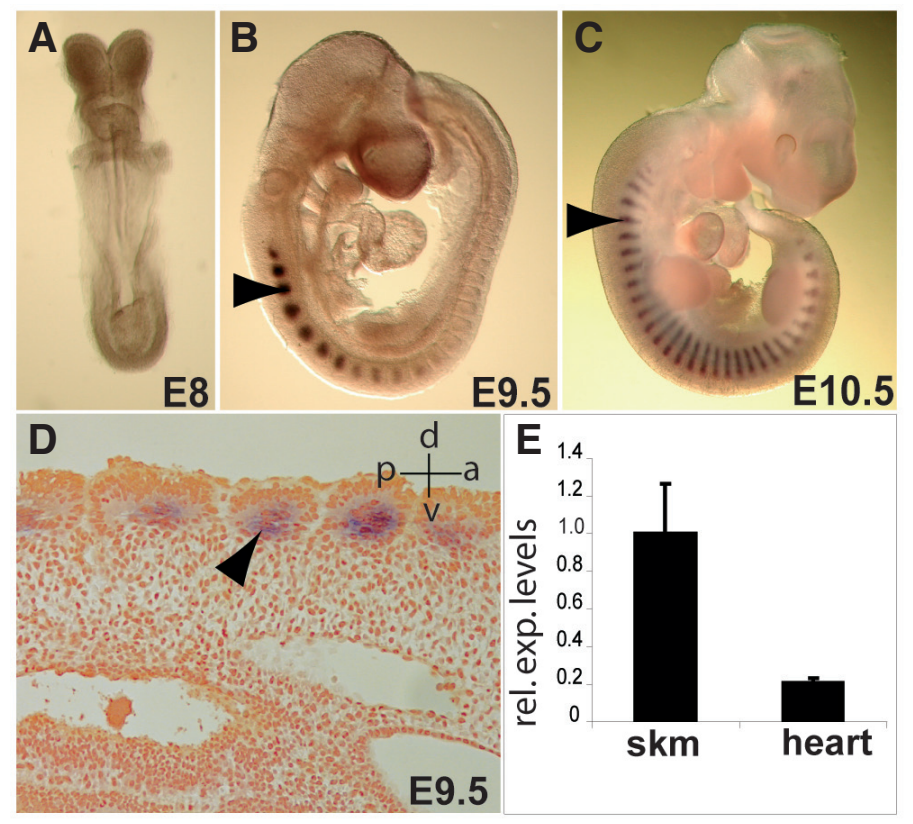

Fig. 1. Sarcosin mRNA expression during mouse embryonic development and in adult heart and skeletal muscle tissue. In situ hybridization with digoxigenin-UTPlabelled specific riboprobe performed on whole-mount mouse embryos at (A) embryonic day E8, (B) E9.5, and (C) E10.5. Sarcosin expression is visible in the somites (arrowheads) from E9.5 onwards. (D) E9.5 embryo subjected to whole-mount in situ hybridization sectioned in the sagittal plane (10 $\mu \mathrm{m}$ thick). Sarcosin expression is visible in the myotome part of the somite (arrowhead). d, dorsal; $v$, ventral; a, anterior; $p$, posterior. (E) Sarcosin transcript levels measured using quantitative RT-PCR in mouse adult skeletal muscle (skm) and heart. Expression levels in skm are set to 1. Values are normalized to the house keeping genes Gapdh, Pgk1 and B-actin. Error bars represent standard error of the mean. 
Confocal imaging at planes above and below the laterally fusing myofibrils revealed that SARCOSIN was not only concentrated between fusing myofibrils but also underneath these structures. SARCOSIN did not colocalize with $\alpha$-ACTININ.

\section{Sarcosin is highly up-regulated in C2C12 myoblast differentiation into myotubes}

C2C12 cells were used to investigate the role of SARCOSIN in skeletal muscle. Sarcosin mRNA levels were readily detectable in undifferentiated myoblasts (day 0), and expression levels were highly up-regulated (approximately 60 fold) in day 6 differentiated myotubes compared to proliferating myoblasts (Fig. 3A). Immunoblot analysis of SARCOSIN protein expression during myoblast differentiation confirmed the results observed with quantitative real-time PCR (Fig. 3B). SARCOSIN protein was present in proliferating myoblasts and expression was up-regulated during differentiation of the myoblasts to myotubes. At the beginning of myoblast differentiaton a down-regulation of SARCOSIN protein was observed

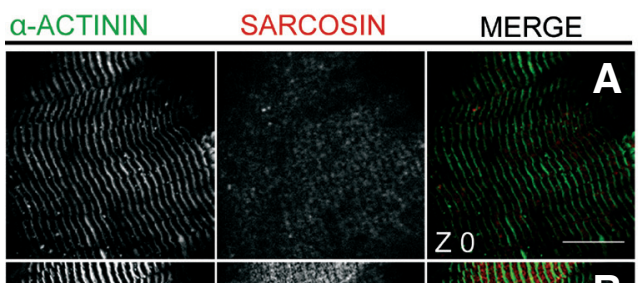

MYOMESIN SARCOSIN MERGE
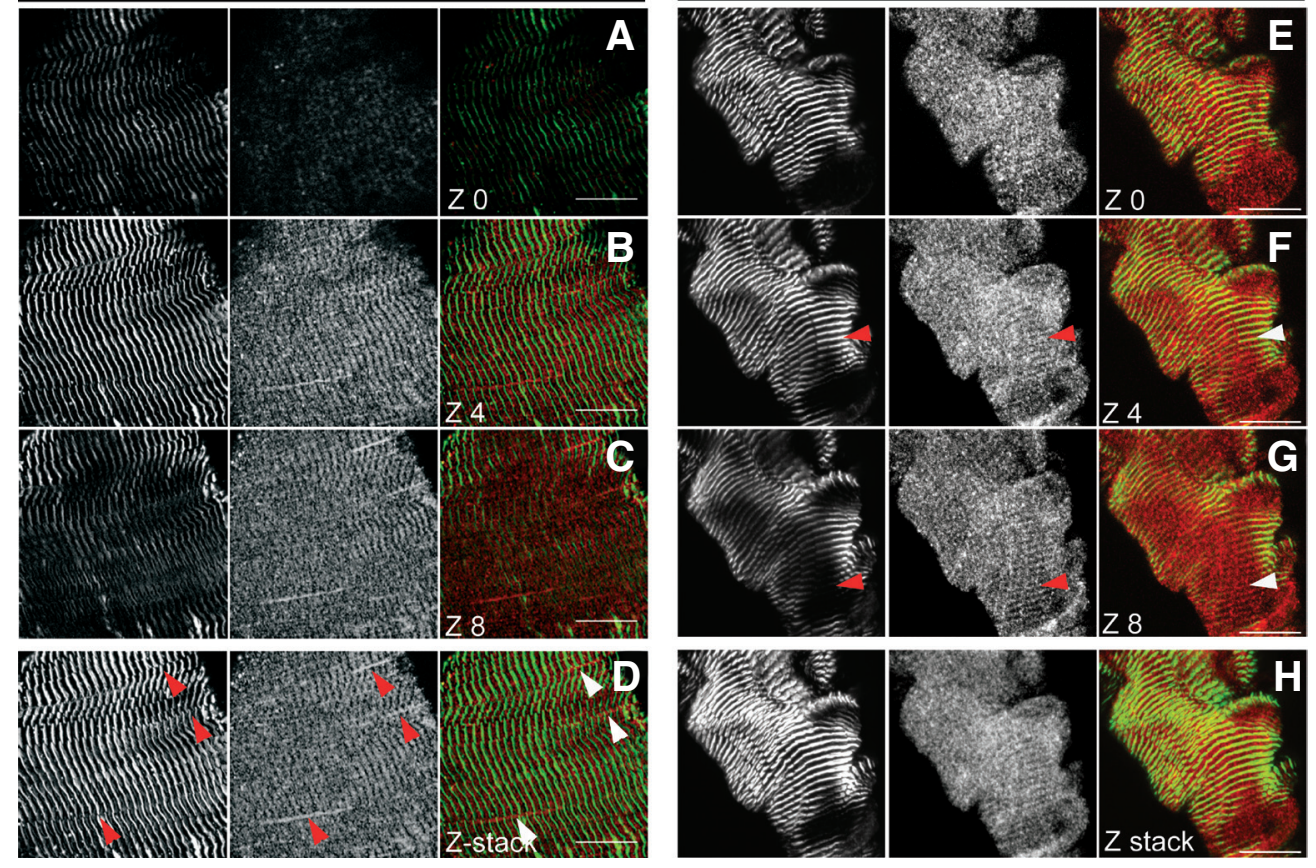

Fig. 2. SARCOSIN expression in adult skeletal muscle. Immunofluorescence staining in adult skeletal muscle tissue. (A-D) $\alpha$-ACTININ (green) and SARCOSIN (red); (A-C) Z0, Z4, Z8: focal planes with a step size of $122 \mathrm{~nm}$ between the individual Z-planes; (D) Z-stack of A-C, arrowheads point to concentrated SARCOSIN staining between (also visible in B) and below (also visible in C) laterally fusing myofibrils. (EH) MYOMESIN (green) and SARCOSIN (red). Arrowheads point to concentrated SARCOSIN staining at the M-band. (E-G) Z0, Z4, Z8: focal planes with a step size of $163 \mathrm{~nm}$ between the individual Z-planes; (H) Z-stack of E-G. Scale bars, $10 \mu \mathrm{m}$.

compared to proliferating myoblasts;

this was not observed at the mRNA level. Immunostaining of differentiating $\mathrm{C} 2 \mathrm{C} 12$ cells revealed expression of SARCOSIN in the cytoplasm of cells that also expressed $\alpha$-ACTININ (Fig. 3C). At day three of differentiation, Zbodies were visible and a colocalization of $\alpha$-ACTININ and SARCOSIN was observed in these Z-bodies. In fused immature myofibrils on day 5 of differentiation SARCOSIN was equally distributed (Fig. 3C).

\section{RNAi mediated knock-down of SARCOSIN expression in C2C12 myoblasts}

In order to establish the function of SARCOSIN in skeletal muscle cells, Sarcosin expression was downregulated in $\mathrm{C} 2 \mathrm{C} 12$ cells. Proliferating $\mathrm{C} 2 \mathrm{C} 12$ myoblasts were transfected with stealth siRNA to Sarcosin or with a mock-control, and $48 \mathrm{hrs}$ after transfection differentiation

Fig. 3. SARCOSIN RNA and protein expression in C2C12 myoblasts differentiating to myotubes. (A) Sarcosin mRNA transcript levels measured using quantitative RT-PCR in C2C12 myoblasts differentiating into myotubes. Values are normalized to the expression of Gapdh, Oaz1 and Rpl22. Error bars represent standard error of the mean. (B) Immunoblot analysis of SARCOSIN, $\alpha$-ACTININ and the housekeeping protein ACTIN in C2C12 myoblasts at days 0, 1, 3 and 6 of differentiation to myotubes. (C) Immunofluorescence staining of $\alpha$-ACTININ (green), SARCOSIN (red) and visualization of nuclei (blue) in differentiating C2C12 cells. Arrowheads point to $Z$ bodies, scale bars $20 \mu \mathrm{m}$.

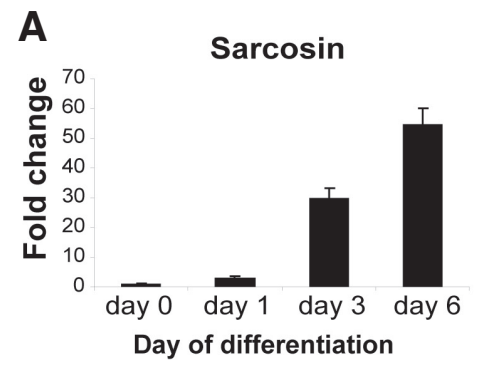

B

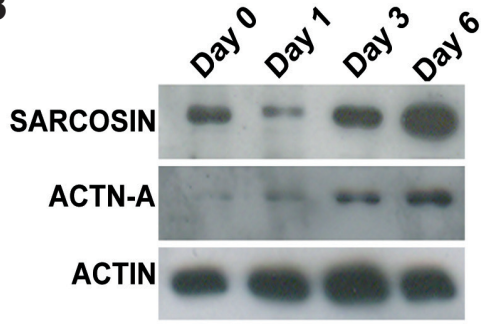

C

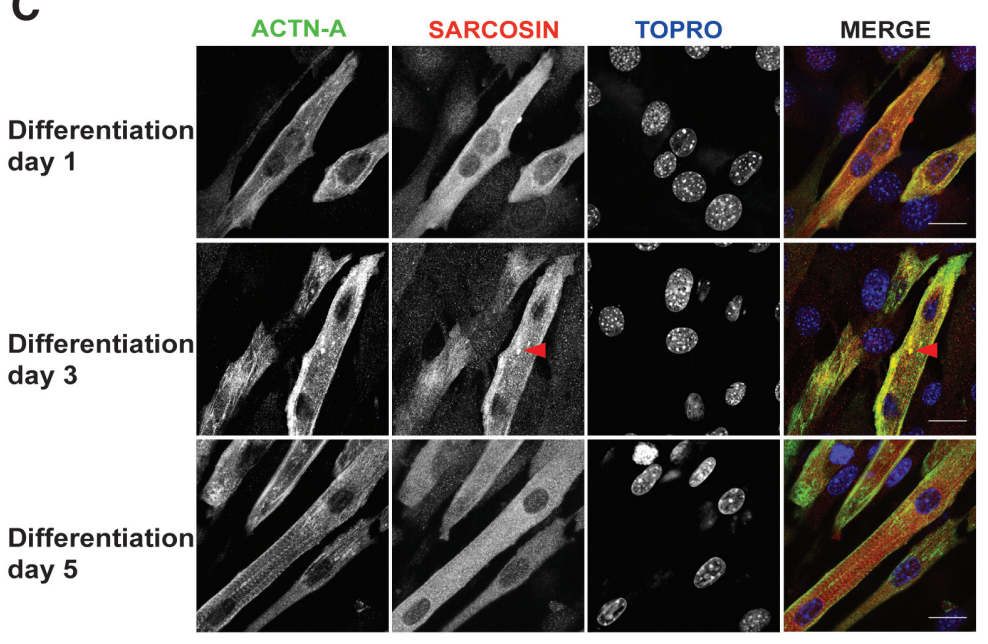


A

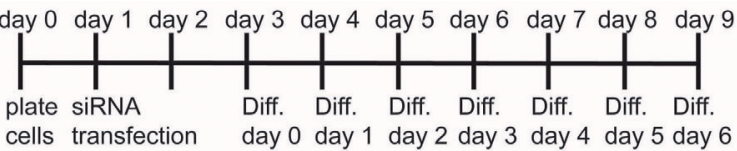

B

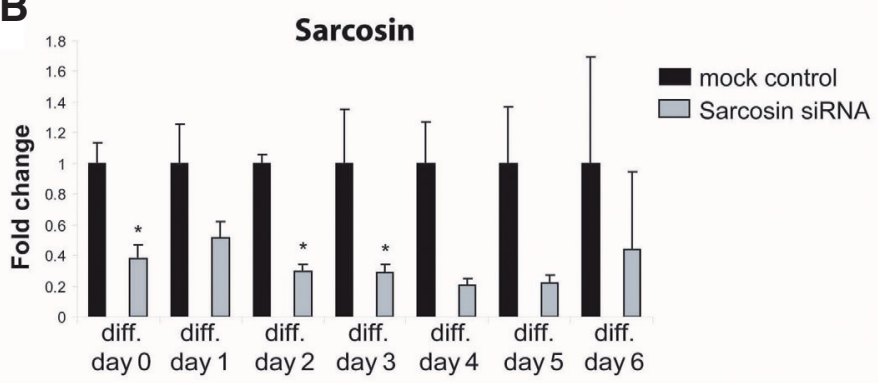

C

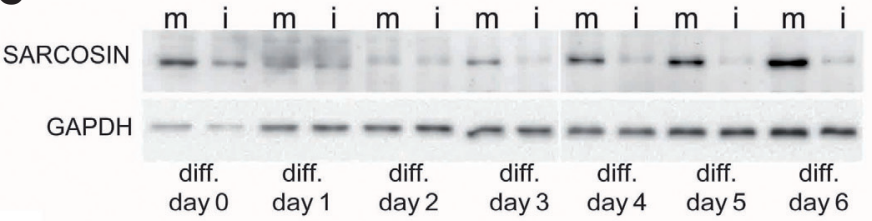

D

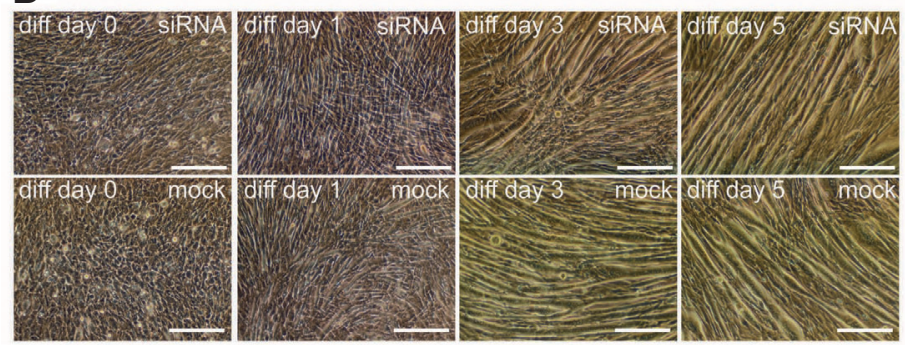

E
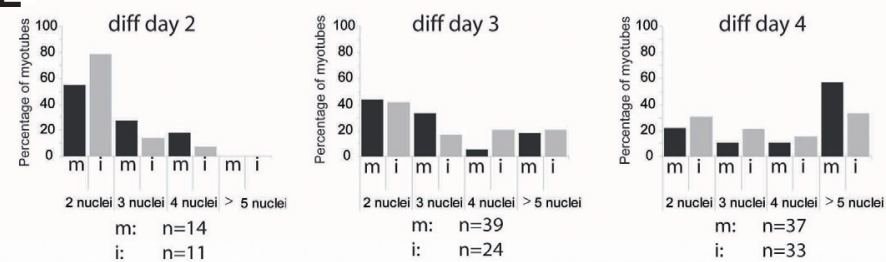

Fig. 4. RNAi mediated knock-down of SARCOSIN expression in C2C12 myoblasts and their differentiation to myotubes. (A) Schematic presentation of the experimental procedure. (B) Sarcosin mRNA transcript levels measured using quantitative real time PCR in C2C12 myoblasts, transfected with Sarcosin siRNA or with mock negative control, differentiating into myotubes. Values are normalized to the housekeeping genes Gapdh, Oaz1 and Rpl22. Expression levels of mock control are set to 1. Error bars represent standard error of the mean, ${ }^{*} p<0.05$ indicates statistical differences between mock control and Sarcosin siRNA on the same day of differentiation. (C) Immunoblot analysis of SARCOSIN expression in C2C12 myoblasts differentiating to myotubes, transfected with siRNA against Sarcosin (i) or with mock negative control (m). Samples collected at days 0 to 6 of differentiation. GAPDH expression was analyzed as loading control. (D) Morphology of myoblasts at different days of differentiation into myotubes, transfected with Sarcosin siRNA or mock control. Scale bars $20 \mu \mathrm{m}$. (E) Quantification of myotube formation by determining cells with multiple nuclei at days 2, 3 and 4 of differentiation in C2C12 cells transfected with Sarcosin siRNA (i) or mock control (m). Shown are combined data from three experiments; $n=$ numbers of myotubes analyzed. was induced by replacing the culture medium with differentiation medium containing $2 \%$ horse serum (Fig. 4A). Sarcosinknock-down was evaluated using quantitative real time PCR and immunoblot analysis. Two days after transfection Sarcosin mRNA levels were decreased to approximately $30 \%$ compared to the mock-control transfected cells and this decrease persisted during at least 8 days after transfection (6 days differentiation) (Fig. 4B). No nonspecific inhibitory effects were observed, in terms of cell death, cell morphology or Sarcosin expression. As expected, a decrease of SARCOSIN protein levels was somewhat delayed when compared to mRNA expression levels. Starting at day 3 of myoblast differentiation and thus 5 days after transfection, SARCOSIN protein levels were substantially decreased in siRNA transfected cells compared to their mock-controls (Fig. 4C). No difference was observed between the morphology of the myotubes in cells transfected with siRNA compared to their mock-control (Fig. 4D). In fact, both in siRNA transfected myoblasts and in the mock-controls spontaneously contracting myotubes were observed. However, when we analyzed myotube fusion by determining the numbers of nuclei per cells in time we observed a small reduction in the percentage of cells with more than 5 nuclei at day 4 of differentiation after Sarcosin knockdown (Fig. 4E) suggesting an inhibition of differentiation. Interestingly, at day 2 of differentiation a small increase in cells with 2 nuclei was observed (Fig. 4E) after Sarcosin knockdown indicating an initial enhanced differentiation. Although this pattern was observed in 3 independent experiments, there was a substantial variation in the numbers of nuclei per myotubes between three independent experiments.

To assess the effects of SARCOSIN knock-down on $\mathrm{C} 2 \mathrm{C} 12$ myoblast differentiation, the expression of genes involved in myofibril function was analyzed by quantitative RT-PCR. Expression levels of Lasp-1 (Fig. 5A), coding for a binding partner of SARCOSIN that is involved in cell-migration (Spence et al., 2006), decreased during differentiation of $\mathrm{C} 2 \mathrm{C} 12$ cells to myotubes. On days 4 and 6 of differentiation, Lasp-1 transcript levels were significantly increased in cells treated with Sarcosin siRNA when compared to the mock-controls, although differences were minor and may not have biological significance.

$\mathrm{N}-\mathrm{RAP}$ is involved in myofibril assembly and was identified as a binding partner of SARCOSIN (Dhume et al., 2006; Lu et al., 2003). Transcript levels of $N$-rap increased considerably (up to 16 times) during myoblast differentiation (Fig. 5B). Between days 2 and 4 of myoblast differentiation $N$-rap transcript levels in siRNA transfected cells were up-regulated compared to their mock-controls and this up-regulation was significant on day 2 of differentiation. $\alpha$ Actinin and Nebulin code for two building blocks of the sarcomeric structures (Clark et al., 2002) and their expression was highly upregulated during myoblast differentiation (Fig. 5 C,D). $\alpha$-Actinin transcript levels were readily detectable in proliferating myoblasts, and transcript levels were up-regulated in Sarcosin siRNA transfected cells compared to their mock-controls between days 2 and 4 of myoblast differentiation, this up-regulation was significant on day 3 of differentiation (Fig. 5C). Nebulin mRNA levels could only be detected reliably as of day 2 of myoblast differentiation and Nebulin transcript levels were up-regulated in siRNA transfected myoblasts compared to their mock controls between day 2 and 4 of myoblast differentiation (Fig. 5D).

RNA expression levels were measured for 3 isoforms of myosin heavy chain (MHC). Embryonic-mhc is expressed in developing 
skeletal muscle and increased during C2C12 myoblast differentiation (Fig. 5E). MHC2B is expressed in fast-twitching muscle and $M h c 2 b$ transcript levels increased up to 12 times during myoblast differentiation (Fig. 5F). Finally, Non-muscle Mhc2b, coding for the myosin present in premyofibril structures before replacement with muscle myosin, decreased during myoblast differentiation (Fig. $5 G$ ). Of these myosins, only Mhc2b transcripts were consistently up-regulated in siRNA treated myoblasts compared to their mock controls.

To examine whether SARCOSIN is involved in muscle atrophy Atrogin mRNA expression levels were measured. No difference in mRNA expression levels were observed between SARCOSIN down-regulated cells and their mock controls (Fig. $5 \mathrm{H}$ ).

Protein expression in differentiating myoblasts in which SARCOSIN was down-regulated by siRNA treatment was analyzed by immunoblotting (Fig. 6A). No major differences when differentiated

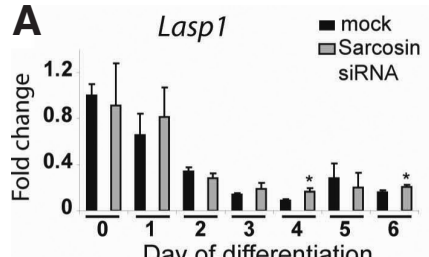

Day of differentiation

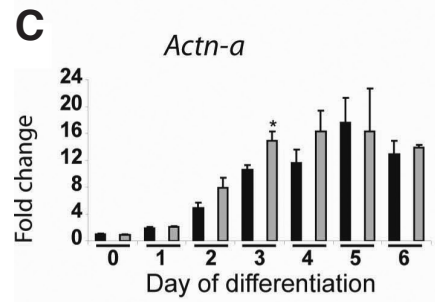

E

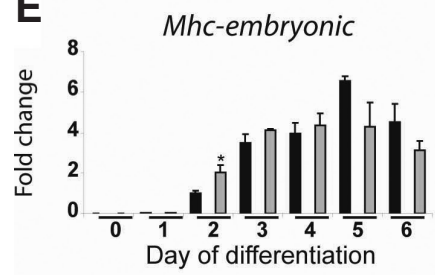

G

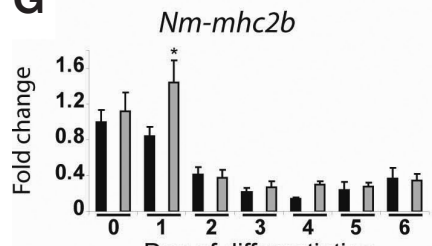

Day of differentiation

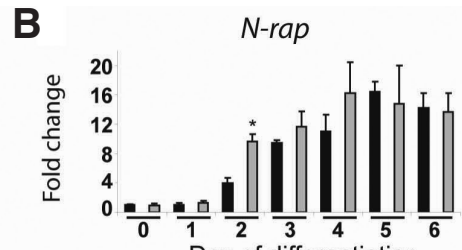

D

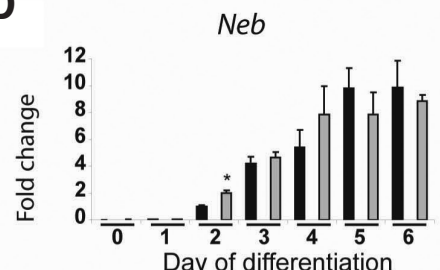

$\mathbf{F}$

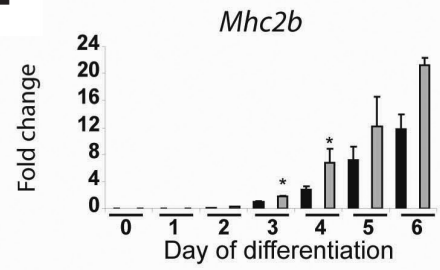

H

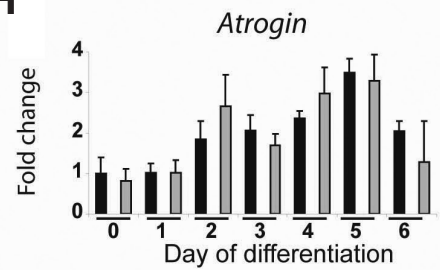

Fig. 5. Effect of SARCOSIN knock-down on mRNA transcript levels of genes involved in C2C12 myoblast differentiation. Messenger RNA transcript levels of (A) Lasp-1; (B) N-rap; (C) $\alpha$-actinin; (D) Nebulin; (E) embryonic myosin heavy chain-embyronic (embryonic $\mathrm{MHC}$ ); (F) MHC2b; (G) non muscle-MHC2b and $\mathbf{( H )}$ Atrogin measured using quantitative real time PCR in C2C12 myoblasts, transfected with stealth siRNA against Sarcosin or with mock negative control, differentiating into myotubes. Experiments were carried out in triplicate. Values are normalized to expression of the housekeeping genes Gapdh, Oaz1 and Rpl22. Errorbars representstandard error of the mean, ${ }^{*} p<0.05$ indicates statistical differences between mock control and Sarcosin siRNA on the same day of differentiation. cells were compared (Fig. 6A). There appeared to be a weak upregulation of $\alpha$-ACTININ and MYOGENIN expression during early differentiation. Although this upregulation was observed in several independent experiments (not shown) the significance is not known. Immunofluorescence analysis of the myotubes in which SARCOSIN was down-regulated did not reveal any disruption or difference in myotube formation as studied by $\alpha$-ACTININ staining (Fig. 6B).

\section{Discussion}

SARCOSIN is a protein exclusively expressed in sarcomeric muscle and is described to play a role in late myofibril assembly in cultured cardiomyocytes. SARCOSIN's role in skeletal myoblast differentiation is unknown (Greenberg et al., 2008; Taylor et al., 1998).

To identify the role of SARCOSIN in skeletal muscle, expression of Sarcosin mRNA during early mouse embryonic development was analyzed. Sarcosin mRNA expression was first observed in the myotome part of mature somites in E9.5 embryos. This late expression during somite development implicates that Sarcosin expression is not necessary for initial somite formation from the paraxial mesoderm. Whether SARCOSIN plays a role in somite epithelialization and somite segmentation into the sclerotome and myotome remains to be determined (for review on somitogenesis see (Kalcheim and Ben-Yair, 2005) and references therein). Remarkably, Sarcosin mRNA was not expressed in the early embryonic heart (E8-E10.5) while at these embryonic stages the heart is contracting and thus functional sarcomeres are present. It is possible that the Sarcosin mRNA levels in the heart are below the detection limit for ISH at these embryonic stages. In adult tissues Sarcosin mRNA was expressed at a lower level in the heart compared to skeletal muscle as was previously demonstrated (Taylor et al., 1998). Knockout studies in mice will help reveal the role of SARCOSIN in somite maturation and in heart development.

SARCOSIN protein is expressed throughout the cytoplasm and is concentrated between laterally fusing Z-discs of nascent myofibrils in cultured cardiomyocytes (Greenberg et al., 2008; Lu et al., 2003). We observed a similar protein localization pattern in mouse adult skeletal muscle. SARCOSIN was also concentrated underneath laterally fusing myofibrils in adult skeletal muscle, and SARCOSIN partially co-localized with the M-band protein MYOMESIN. This expression pattern suggests that SARCOSIN has a function in the lateral fusion of myofibrils in skeletal muscle. A similar function for SARCOSIN in cultured cardiomyocytes has been reported (Greenberg et al., 2008). In differentiating C2C12 cells we observed SARCOSIN distributed rather evenly throughout the cytoplasm which indeed has been described very recently (Paxton et al., 2011), suggesting different functions for SARCOSIN in developing and adult muscle cells.

In proliferating C2C12 myoblasts SARCOSIN was already expressed at considerable levels, which is in contrast to results from Paxton et al., (2011) who observed absence of SARCOSIN (Krp1) expression in proliferating $\mathrm{C} 2$ cells. This could indicate that in our experiments some cells were already differentiating in the growth medium. On the other hand, the observed initial downregulation of SARCOSIN during the early phases of directed differentiation suggests different roles for SARCOSIN in proliferating cells versus its function in differentiating cells. Paxton and colleagues further reported that SARCOSIN was only expressed in cells that also expressed MYOGENIN, and suggested that MYOGENIN expres- 
sion in fact precedes SARCOSIN expression (Paxton et al., 2011). In our experiments however SARCOSIN expression was observed even in the absence of MYOGENIN expression, which argues against such a hierarchy. Paxton and coworkers observed that overexpression of SARCOSIN in differentiating C2 cells reduces cell numbers, but whether this reduction also occurred in proliferating cells in growth medium is not known (Paxton et al., 2011).

In this study, protein expression was detected with immunoblot analysis. It is possible that because of the method used the protein levels in the samples do not reflect the total amount of proteins in the cells particularly for cytoskeletal elements and myofibrils. However, both $\alpha$-ACTININ (Fig. 3B) and MYOSIN (Fig. 6A) were detected using immunoblot analysis. Since all cell lysate samples were treated similarly, we are of the opinion that the different samples can be mutually compared. Indeed protein levels of $\beta$-ACTIN as detected with immunoblot analysis were similar between samples where similar amounts of protein were analyzed (Fig. 3B). In addition, immunoblots with lysed samples that were not centrifuged before electrophoresis yielded similar results (data not shown).

To test whether SARCOSIN functions in myoblast differentiation

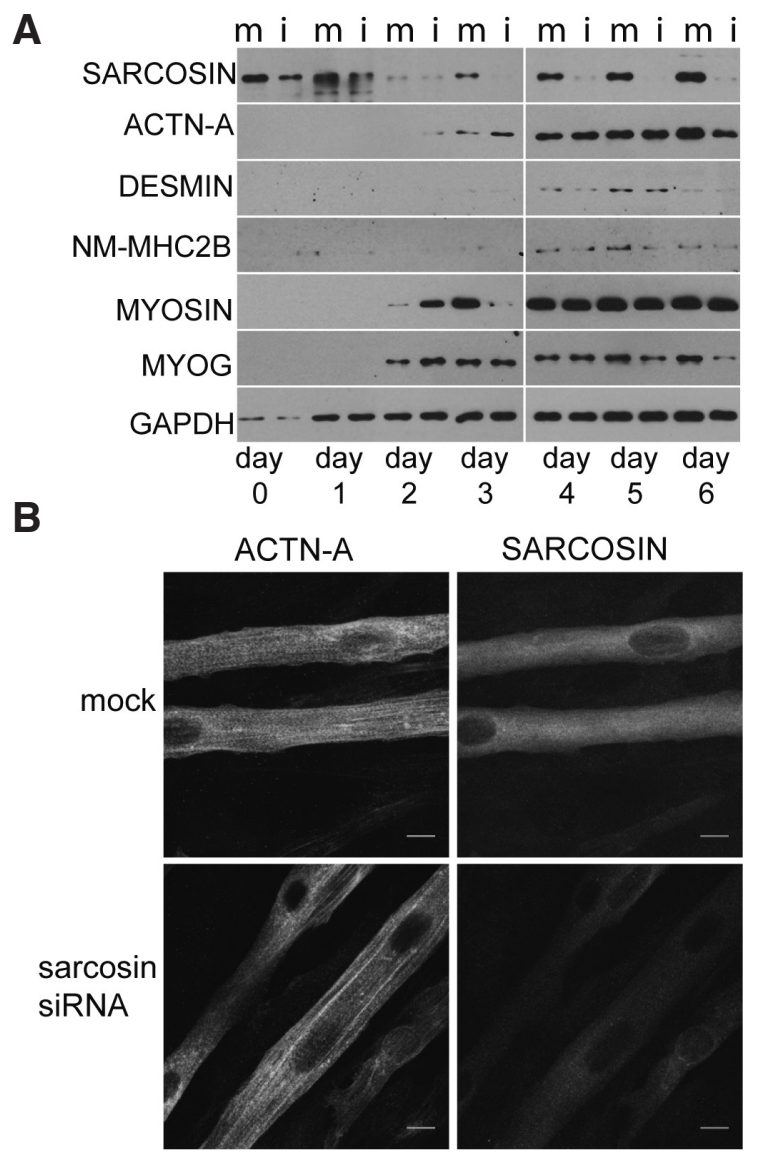

Fig. 6. Effect of SARCOSIN knock-down on proteins involved in C2C12 myoblast differentiation. (A) Immunoblot analysis of SARCOSIN, $\alpha$ ACTININ, DESMIN, non muscle myosin heavy chain 2B (NM-MHC2B), all sarcomeric myosin (MYOSIN), MYOGENIN and GAPDH in C2C12 myoblasts, transfected with stealth siRNA against Sarcosin (i) or with mock negative control ( $m$ ), differentiating into myotubes (day 0-6: differentiation day 0-6). (B) Immunofluorescence staining of $\alpha$-ACTININ (left) and SARCOSIN (right) in myotubes, transfected with either mock control (top) or Sarcosin SiRNA (bottom); scale bars $10 \mu \mathrm{m}$. and fusion, SARCOSIN expression levels were down-regulated in C2C12 cells using siRNA. During differentiation, RNA expression levels of sarcomeric proteins and binding partners of SARCOSIN were measured using quantitative real-time PCR in siRNA treated cells and compared to their mock controls. During skeletal muscle differentiation the mRNAlevels of $N$-rap, $\alpha$-actinin, Nebulin, Embryonic-mhc and Mhc2b were highly up-regulated. RNA expression levels from these sarcomeric proteins were higher in the siRNA treated cells compared to their mock controls starting from day 2 of differentiation, suggesting that knock-down of SARCOSIN affected differentiation. In agreement with this, $\alpha$-ACTININ, MYOSIN and MYOGENIN protein levels were slightly increased at day 2 of differentiation compared to their mock controls. Indeed at day 2 of differentiation we observed more $\mathrm{C} 2 \mathrm{C} 12$ cells with 2 nuclei after Sarcosinknockdown, indicating a weak enhanced early differentiation. However, SARCOSIN is not essential for myotobe differentiation since we did observe spontaneously contracting myotubes after differentiation of $\mathrm{C} 2 \mathrm{C} 12$ cells in which Sarcosin expression was downregulated. Also, no difference in $\alpha$-ACTININ localization was observed in differentiating $\mathrm{C} 2 \mathrm{C} 12$ cells with downregulated Sarcosin expression (Fig. 6B).

Promoter analysis studies have revealed two pairs of E-boxes upstream of the Sarcosincoding sequence, suggesting that Sarcosin is a myogenic target (Paxton etal., 2011). Based on its expression in differentiating $\mathrm{C} 2$ cells, combined with knockdown and overexpression experiments it has been suggested that SARCOSIN (Krp1) has functions in myogenesis before myofibrillar formation (Paxton et al., 2011). The localised expression that we observed in mature muscle cells suggests an additional function in mature myofibers.

Very recently it has been described that both knockdown and overexpression of Sarcosin (Krp1) resulted in reduced myoblast differentiation (Paxton et al., 2011). We found similar results as we observed a reduced percentage of cells with more than 5 nuclei after Sarcosin knockdown in C2C12 cells. Taken together, it appears that Sarcosin at the first phases of myoblast differentiation has an inhibitory function, while at later stages of differentiation it has a stimulatory action. This could explain the paradoxical inhibition of differentiation observed after both knockdown and overexpression of Sarcosin (Paxton et al., 2011). Although Sarcosin expression is highly upregulated in differentiating skeletal muscle cells, its presence does not seem to be essential for the differentiation process itself. Rather, it might be that SARCOSIN is important for the correct functioning of skeletal muscle cells, as suggested by the expression of SARCOSIN in adult skeletal muscle.

\section{Materials and Methods}

\section{Animals}

C57BI/6 mice were intercrossed and the time of feritilization was taken as the midpoint of the darkcycle before the copulation plug was found. Females were sacrificed for collection of embryos at various time points. Embryos were fixed in 4\% (w/v) paraformaldehyde (PFA) (Electron Microscopy Science, Hatfield, PA) in phosphate buffered saline (PBS, Braun, Melsungen, Germany) at $4^{\circ} \mathrm{C}$ overnight and stored in $100 \%$ methanol at $-20^{\circ} \mathrm{C}$. All animal handling procedures were approved by the institutional animal experiments committee.

\section{Whole-mount in situ hybridization}

Whole-mount in situ hybridization (ISH) was performed as described previously (Wilkinson, 1992). For probe preparation, purified PCR product was generated to use as a template, with Sarcosin-specific primers (forward: 
CCTTACCCAGGTTCCTCACA, reverse: CCAATCTTAGGATGGCCAGA) with the T3 promoter sequence (ATACAATTAACCCTCACTAAAGGG) at the 5'end of the forward primer and the T7 promoter sequence (ATAGGTAATACGACTCACTATAGGGC) at the 3'end of the reverse primer. RNA probes were synthesized using $T 7$ (antisense) polymerase and DIG labelled nucleotides according to the manufacturer's protocol (Roche Applied Sciences, Almere, The Netherlands). Embryos were imaged using a Zeiss Axioplan SZX9 coupled to a Leica DFC480 digital camera.

\section{Cell culture and transfection}

The murine myoblast cell line C2C12 (ATCC CRL-1772) was cultured at $37^{\circ} \mathrm{C}, 5 \% \mathrm{CO}_{2}$ in Dulbecco's Modified Eagle's Medium (DMEM-HG, Invitrogen, Carlsbad, CA) based proliferation medium supplemented with $10 \%$ fetal bovine serum (FBS, Invitrogen), $1 \times$ non-essential amino acids (Invitrogen) and $50 \mathrm{U} / \mathrm{ml}$ penicillin and $50 \mu \mathrm{g} / \mathrm{ml}$ streptomyocin (Invitrogen). Myotube formation was induced by replacing FBS in the culture medium with $2 \%$ horse serum (Differentiation medium, Vector Laboratories, Burlingame, CA). To reduce endogenous SARCOSIN expression, cells were transfected with stealth siRNAduplex oligoribonucleotides against Sarcosin (5'-CCACTGAAGTCAATGACATATGGAA- 3') (Invitrogen) using Lipofectamine 2000 according to the manufacturer's protocol (Invitrogen). Universal control siRNAs (Invitrogen) with GC percentage similar to the Sarcosin stealth siRNA were used as a negative control. This is referred to as 'mock control' or 'mock' throughout the manuscript. Briefly, one day before transfection cells were plated at a density of 4000 cells $/ \mathrm{cm}^{2}$ on gelatin-coated dishes in proliferation medium. Cells were transfected with $40 \mathrm{pmol} / \mathrm{ml}$ Sarcosin siRNA and medium was changed $8 \mathrm{hrs}$ after transfection. Differentiation was induced $48 \mathrm{hrs}$ after transfection.

\section{RNA isolation and quantitative real- time PCR}

Total RNA of $\mathrm{C} 2 \mathrm{C} 12$ cells was extracted using the RNeasy minikit (Qiagen, Valencia, CA) and on-column DNAse treatment (Qiagen) was performed. Total RNA from adult mouse heart and skeletal muscle was isolated using Trizol reagent (Invitrogen). Total RNA was reverse transcribed using Superscript II reverse-transcription polymerase (Invitrogen) and random primers (Invitrogen) according to the supplier's protocol.

For quantitative real-time PCR, each reaction contained $10 \mu \mathrm{l}$ SYBRGreen Mastermix (BIO-RAD, Hercules, CA), $0.5 \mathrm{mM}$ forward primer, 0.5 $\mathrm{mM}$ reverse primer and $1 \mu \mathrm{l} \mathrm{cDNA}$ as a template in a final volume of 20 $\mu \mathrm{l}$. Primers were designed using Primer 3 software (Rozen and Skaletsky, 2000) or based on literature (Table 1) (de Jonge et al., 2007; Dhume et al., 2006; Martinez-Fernandez et al., 2006; Sakiyama et al., 2005; Sultan et al., 2006; Willems et al., 2006). Reactions were carried out in duplicate for each sample. Negative controls were reactions containing template synthesized without reverse transcriptase and reactions containing $\mathrm{H}_{2} \mathrm{O}$ instead of cDNA. The reactions were amplified on a MyIQ Real-Time PCR detection system (BIO-RAD) using the following program: initial denaturation at $95^{\circ} \mathrm{C}$ for $3 \mathrm{~min}$, followed by 40 cycles of $30 \mathrm{sec}$ at $95^{\circ} \mathrm{C}, 30 \mathrm{sec}$ at the corresponding annealing temperature (Table 1) and $30 \mathrm{sec}$ at $72^{\circ} \mathrm{C}$. Melting curves were produced to verify single PCR product amplification. Efficiency of the PCR reactions was tested using standard curves synthesized from either a dilution series of CDNA or PCR product. PCR products were separated on $1 \%$ agarose gels against a $100 \mathrm{bp}$ ladder and visualized with ethidium bromide. To verify the specificity, the PCR products were

TABLE 1

PRIMERS USED FOR RT-PCR AND Q-PCR

\begin{tabular}{|c|c|c|c|c|c|}
\hline Gene & 5 ' forward primer & 5 ' reverse primer & $\mathrm{Ta}\left({ }^{\circ} \mathrm{C}\right)$ & Amplicon size (bp) & Reference \\
\hline Gapdh & CGTGCCGCCTGGAGAAAC & TGGGAGTTGCTGTTGAAGTCG & 56 & 136 & Beacon designer \\
\hline Oaz1 & GGATCCTCAATAGCCACTGC & TACAGCAGTGGAGGGAGACC & 58 & 150 & de Jonge et al., 2007 \\
\hline Rpl22 & CTGGGCTGCTGCTCCTTTG & AATCACCTGTCTGCTTCTGAGG & 58 & 250 & de Jonge et al., 2007 \\
\hline Pgk1 & CTCCGCTTTCATGTAGAGGAAG & GACATCTCCTAGTTTGGACAGTG & 58 & 117 & Willems et al., 2006 \\
\hline B-actin & GACATCCGTAAAGACCTCTATG & АСTCCTGCTTGCTGATCC & 58 & 223 & Beacon designer \\
\hline Sarcosin & CCTAGCAACGCGCTTAAATC & GAAACTGCGTGAATCCCTGT & 62 & 161 & PRIMER 3 \\
\hline Nebulin & АСТGТССТСТАТСССАТСАС & GCCATACATCCAGCCTTCAT & 60 & 151 & Dhume et al., 2006 \\
\hline$N$-rap & AAACCAACGCAGCCAACCTG & CGTGCCTGTAGAGAAAGTCA & 60 & 150 & Dhume et al., 2006 \\
\hline Alfa-actinin(2+3) & ATCATCCTCCGCTTCGCCATTC & TCTTCAGCATCCAACATCTTAGG & 58 & 290 & Dhume et al., 2006 \\
\hline Lasp1 & GCCTGCAGTGAAGGGTAGAG & GACACGACGACAGAAAAGCA & 58 & 249 & PRIMER 3 \\
\hline MHC-embryonic & GCCAGGATGGGAAAGTCACTGTGG & GGGCTCGTTCAGGTGGGTCAGC & 62 & 137 & Martinez-Fernandez et al., 2006 \\
\hline MHC2B & ACAGACTAAAGTGAAAGCC & СТСTCAACAGAAAGATGGAT & 54 & 215 & Sakiyama et al., 2005 \\
\hline NM-MHC2B & TGTCATCTACAACСCTGCСАСТC & GCTTTCTTCCСАTTCTCTGCCAG & 58 & 150 & Dhume et al., 2006 \\
\hline Atrogin & СTCTGTACCATGCCGTTCCT & GGCTGCTGAACAGATTCTCC & 55 & 172 & Sultan et al., 2005 \\
\hline
\end{tabular}

TABLE 2

ANTIBODIES

\begin{tabular}{|c|c|c|c|}
\hline Protein & Manufacturer (Product code) & Immuno blot (IB)/Immunofluorescence (IF) & Description \\
\hline SARCOSIN & aviva systems biology (ARP38732) & 1 to $1600(\mathrm{IB})$ & rabbit polyclonal \\
\hline SARCOSIN & generous gift of Prof. Bradford Ozanne & 1 to 500 (IF) & rabbit polyclonal \\
\hline GAPDH & SIGMA (G8795) & 1 to 20000 (IB) & mouse monoclonal \\
\hline ACTIN & Chemicon (MAB1501, clone C4) & 1 to $1000(\mathrm{IB})$ & mouse monoclonal \\
\hline$\alpha$-ACTININ & SIGMA (A7811) & 1 to 2500 (IB) / 1 to 500 (IF) & mouse monoclonal \\
\hline MYOGENIN & BD Pharmingen (556358) & 1 to 200 (IF) & mouse monoclonal \\
\hline MYOSIN (all sarcomeric) & Development studies hybridoma bank (MF20) & 1 to $1000(\mathrm{IB})$ & mouse monoclonal \\
\hline NON MUSCLE MYOSIN & Development studies hybridoma bank (CMII23) & 1 to 500 (IB) & mouse monoclonal \\
\hline DESMIN & DakoCytomation (M0760) & 1 to 200 (IB) & mouse monoclonal \\
\hline MYOMESIN & generous gift of Dr Elisabeth Ehler & 1 to $10(\mathrm{IF})$ & mouse monoclonal \\
\hline
\end{tabular}


sequenced. Data were analyzed with iQ5 software (BIO-RAD). Starting quantities of all genes were calculated based on their standard curves. Using ge-Norm, gene expression normalization factors for each sample were calculated based on the expression levels of the reference genes glyceraldehyde-3-phosphate dehydrogenase (Gapdh), phosphoglycerate kinase 1 (Pgk1), and beta-actin (B-actin) for data in Fig. 1, and to the expression levels of Ornithine decarboxylase antizyme 1 (Oaz1), ribosomal protein I22 (Rp/22) and Gapdh for data in Figs. 3-5. The underlying principles and calculations used are described in (Vandesompele et al., 2002). Each experiment was performed 3 times independently (biological replicates) and gene expression detection of each sample was performed twice (technical replicate). Statistical differences between expression levels were tested using a nonparametric Mann-Whitney test. Differences were considered significant at $\mathrm{P}<0.05$.

\section{Protein isolation and immunoblotting}

To prepare protein cell extracts $\mathrm{C} 2 \mathrm{C} 12$ cells were washed with ice cold PBS and lysed in buffer containing $140 \mathrm{mM} \mathrm{NaCl}, 50 \mathrm{mM}$ Tris-HCL pH7.5, $1 \mathrm{mM}$ EDTA, $0.1 \%$ Triton X-100, 10\% Glycerol and protease inhibitor cocktail ( 1 tablet per $50 \mathrm{ml}$ lysis buffer) (Roche Applied Sciences). Cell lysates were clarified by centrifugation at $10,000 \mathrm{rpm}$ and $4^{\circ} \mathrm{C}$ for $15 \mathrm{~min}$ and the supernatants protein contents were measured using a DC-protein assay (BIO-RAD). Equal concentrations of protein were suspended in Laemmli's sodium dodecylsulfate-(SDS) sample buffer. Protein samples were separated by SDS-polyacrylamide gel electrophoresis and transferred to Trans-Blot nitrocellulose transfer membrane (BIO-RAD). Membranes were blocked using 5\% Blotting Grade Blocker non-fat dry milk (BIO-RAD) in $1 \mathrm{x}$ Tris Buffered Saline-0.1\% Tween (TBST) for $1 \mathrm{hr}$ at room temperature (RT) and subsequently probed with primary antibody (Table 2) overnight at $4^{\circ} \mathrm{C}$. Next the membranes were incubated with 1 to 10,000 dilution of either horseradish peroxidase-conjugated goat anti-rabbit IgG or goat anti-mouse IgG (Santa Cruz Biotechnology, Santa Cruz, CA) for $1 \mathrm{hr}$ at RT. Signals were visualized using SuperSignal West Dura Extended Duration Substrate (Pierce, ThermoScientific, Erembodegem, Belgium) and exposure to X-ray film (Fuji, Dusseldorf, Germany) or detected with a ChemiDoc XRS system (BIO-RAD).

\section{Immunofluorescence staining and microscopy}

C2C12 cells cultured on gelatine-coated glass cover slips and frozen tissue sections of mouse skeletal muscle and heart tissue were fixed in 4\% PFA for 10 min at RT. C2C12 cells were permeabilized after fixation for 8 min using $0.1 \%$ Triton X100 in PBS. Slides were blocked in PBS-0.1\% Tween 20 containing 4\% normal goat serum (PBST) for $1 \mathrm{hr}$ at RT. For staining cells were incubated overnight at $4^{\circ} \mathrm{C}$ with primary antibody (Table 2), and subsequently incubated for $1 \mathrm{hr}$ at RT with secondary antibodies: goat anti-mouse Alexa488-conjugated and goat anti-rabbit Alexa568conjugated (1:200, Invitrogen). The antibodies were diluted in PBST and rabbit and mouse isotypes served as negative control. Slides were mounted in Fluosave mounting medium (Calbiochem, Merck Chemicals Ltd, Nothingham, UK) and analyzed by normal fluorescence or confocal laser scanning microscopy.

\section{Myotube quantification}

Myotubes were visualized using $\alpha$-actining immunofluorescent staining while the nuclei were stained using Topro. For each differentiation day and for both the mock control and siRNA treated cells myotubes in 6 microscopic fields were scored in a blinded fashion for the number of nuclei present in each myotube.

\section{Acknowledgements}

This work was supported by a SenterNovem grant from the Ministry of Economic Affairs. The authors would like to thank Anko M. de Graaff and Richard W. Wubbolts of the Center for Cell Imaging at the Faculty of Veterinary Medicine in Utrecht for assistance in confocal laser scanning microscopy. We thank ProfBradford W. Ozanne (Beatson Institute for Cancer research, Glasgow, UK) for the generous gift of the Sarcosin antibody and Dr Elisabeth Ehler (Kings College, London, UK) for the Myomesin antibody.

\section{References}

ADAMS J, KELSO R, COOLEY L (2000). The kelch repeat superfamily of proteins propellers of cell function. Trends Cell Biol 10: 17-24.

BANG ML, GREGORIO C, LABEIT S (2002). Molecular dissection of the interaction of desmin with the C-terminal region of nebulin. J Struct Biol 137: 119-27.

BUCKINGHAM M (2001). Skeletal muscle formation in vertebrates. Curr Opin Genet Dev 11: 440-448.

CHEW CS, CHEN X, PARENTE JA JR, TARRER S, OKAMOTO C, QIN HY (2002). Lasp-1 binds to non-muscle F-actin in vitro and is localized within multiple sites of dynamic actin assembly in vivo. J Cell Sci 115: 4787-4799.

CLARK KA, MCELHINNY AS, BECKERLE MC, GREGORIO CC (2002). Striated muscle cytoarchitecture: an intricate web of form and function. Annu Rev Cell Dev Biol 18: 637-706.

DE JONGE HJ, FEHRMANN RS, DE BONT ES, HOFSTRA RM, GERBENS F, KAMPS WA, DE VRIES EG, VAN DER ZEE AG, TE MEERMAN GJ, TER ELST A (2007). Evidence based selection of housekeeping genes. PLoS ONE 2:e898.

DHUME A, LU S, HOROWITS R (2006). Targeted disruption of N-RAP gene function by RNA interference: a role for N-RAP in myofibril organization. Cell Motil Cytoskeleton 63: 493-511

GRAY CH, MCGARRY LC, SPENCE HJ, RIBOLDI-TUNNICLIFFE A, OZANNE BW (2009). Novel beta-propeller of the BTB-Kelch protein Krp1 provides a binding site for Lasp-1 that is necessary for pseudopodial extension. J Biol Chem 284 30498-30507.

GREENBERG CC, CONELLY PS, DANIELS MP, HOROWITS R (2008). Krp1 (Sarcosin) promotes lateral fusion of myofibril assembly intermediates in cultured mouse cardiomyocytes. Exp Cell Res 314: 1177-1191.

HERRERAAH, ELZEY B, LAW DJ, HOROWITS R (2000). Terminal regions of mouse nebulin: sequence analysis and complementary localization with N-RAP. Cell Motil Cytoskeleton 45: 211-222

KALCHEIM C, BEN-YAIR R (2005). Cell rearrangements during development of the somite and its derivatives. Curr Opin Genet Dev 15: 371-380.

LIN YH, PARK ZY, LIN D, BRAHMBHATT AA, RIO MC, YATES JR 3RD, KLEMKE $R L$ (2004). Regulation of cell migration and survival by focal adhesion targeting of Lasp-1. J Cell Biol 165: 421-432.

LU S, BORST DE, HOROWITS R (2005). N-RAP expression during mouse heart development. Dev Dyn 233: 201-212.

LU S, CARROLL SL, HERRERA AH, OZANNE B, HOROWITS R (2003). New N-RAP-binding partners alpha-actinin, filamin and Krp1 detected by yeast twohybrid screening: implications for myofibril assembly. J Cell Sci 116: 2169-2178.

US, BORST DE, HOROWITS R (2008). Expression and alternative splicing of N-RAP during mouse skeletal muscle development. Cell Motil Cytoskeleton 65: 945-954

MARTINEZ-FERNANDEZ S, HERNANDEZ-TORRES F, FRANCO D, LYONS GE, NAVARROF,ARANEGAAE (2006). Pitx2c overexpression promotes cell proliferation and arrests differentiation in myoblasts. Dev Dyn 235: 2930-2939.

NEUHAUS P, JASCHINSKY B, SCHNEIDER S, NEUHAUS H, WOLTERS A, EBELT $\mathrm{H}$, BRAUN T (2006). Overexpression of Kelch domain containing-2 (mKlhdc2) inhibits differentiation and directed migration of $\mathrm{C} 2 \mathrm{C} 12$ myoblasts. Exp Cell Res 312: 3049-3059.

PAXTON CW, COSGROVE RA, DROZD AC, WIGGINS EL, WOODHOUSE S, WATSON RA, SPENCE HJ, OZANNE BW, PELL JM (2011). The BTB-Kelch protein Krp1 regulates proliferation and differentiation of myoblasts. Am J Physiol Cell Physiol 300:C1245-1255.

ROZEN S, SKALETSKY H (2000). Primer3 on the WWW for general users and for biologist programmers. Methods Mol Biol 132: 365-386.

SAKIYAMA K, ABE S, TAMATSU Y, IDE Y (2005). Effects of stretching stress on the muscle contraction proteins of skeletal muscle myoblasts. Biomed Res 26: 61-68.

SCHREIBER V, MASSON R, LINARES JL, MATTEI MG, TOMASETTO C, RIO MC (1998a). Chromosomal assignment and expression pattern of the murine Lasp-1 gene. Gene 207: 171-175.

SCHREIBER V, MOOG-LUTZC, REGNIERCH, CHENARD MP, BOEUFH, VONESCH 
JL, TOMASETTO C, RIO MC (1998b). Lasp-1, a novel type of actin-binding protein accumulating in cell membrane extensions. Mol Med 4: 675-687.

SPENCE HJ, JOHNSTON I, EWART K, BUCHANAN SJ, FITZGERALD U, OZANNE BW (2000). Krp1, a novel kelch related protein that is involved in pseudopod elongation in transformed cells. Oncogene 19: 1266-1276.

SPENCE HJ, MCGARRY L, CHEW CS, CARRAGHER NO, SCOTT-CARRAGHER LA, YUAN Z, CROFT DR, OLSON MF, FRAME M, OZANNE BW (2006). AP-1 differentially expressed proteins Krp1 and fibronectin cooperatively enhance RhoROCK-independent mesenchymal invasion by altering the function, localization, and activity of nondifferentially expressed proteins. Mol Cell Biol 26: 1480-1495.

SULTAN KR, HENKEL B, TERLOU M, HAAGSMAN HP (2006). Quantification of hormone-induced atrophy of large myotubes from C2C12 and L6 cells: atrophy-inducible and atrophy-resistant C2C12 myotubes. Am J Physiol Cell Physiol 290:C650-659.

TAYLORA, OBHOLZK, LINDEN G, SADIEV S, KLAUS S, CARLSON KD (1998). DNA sequence and muscle-specific expression of human sarcosin transcripts. Mol Cell Biochem 183: 105-112.

TOMASETTP C, MOOG-LUTZ C, REGNIER CH, SCHREIBER V, BASSET P, RIO MC
(1995). Lasp-1 (MLN 50) defines a new LIM protein subfamily characterized by the association of LIM and SH3 domains. FEBS Lett 373: 245-249.

VANDESOMPELE J, DE PRETER K, PATTYN F, POPPE B, VAN ROY N, DE PAEPE A, SPELEMAN F (2002). Accurate normalization of real-time quantitative RT-PCR data by geometric averaging of multiple internal control genes. Genome Biol 3:RESEARCH0034.

WILKINSON DG (1992). In situ hybridization: a practical approach. Oxford University Press, USA.

WILLEMS E, MATEIZEL I, KEMP C, CAUFFMAN G, SERMON K, LEYNS L (2006) Selection of reference genes in mouse embryos and in differentiating human and mouse ES cells. Int J Dev Biol 50: 627-635.

WITT CC, BURKART C, LABEIT D, MCNABB M, WU Y, GRANZIER H, LABEIT S (2006). Nebulin regulates thin filament length, contractility, and Z-disk structure in vivo. Embo J 25: 3843-3855.

ZHANG JQ, ELZEY B, WILLIAMS G, LU S, LAW DJ, HOROWITS R (2001). Ultrastructural and biochemical localization of N-RAP at the interface between myofibrils and intercalated disks in the mouse heart. Biochemistry 40: 14898-14906. 


\section{Further Related Reading, published previously in the Int. J. Dev. Biol.}

See our recent Special Issue Angiogenesis in Development \& Cancer edited by Domenico Ribatti and Valentin Djonov at: http://www.ijdb.ehu.es/web/contents.php?vol=55\&issue=4-5

Visualization, characterization and modulation of calcium signaling during the development of slow muscle cells in intact zebrafish embryos

Chris Y. Cheung, Sarah E. Webb, Donald R. Love and Andrew L. Miller

Int. J. Dev. Biol. (2011) 55: 153-174

Sox17-dependent gene expression and early heart and gut development in Sox17-deficient mouse embryos

Sabine Pfister, Vanessa J. Jones, Melinda Power, Germaine L. Truisi, Poh-Lynn Khoo, Kirsten A. Steiner, Masami Kanai-Azuma, Yoshiakira Kanai, Patrick P. L. Tam and David A. F. Loebel

Int. J. Dev. Biol. (2011) 55: 45-58

A conserved MRF4 promoter drives transgenic expression in Xenopus embryonic somites and adult muscle

Timothy J. Hinterberger

Int. J. Dev. Biol. (2010) 54: 617-625

Structure and functions of powerful transactivators: VP16, MyoD and FoxA

Hiroyuki Hirai, Tetsuya Tani and Nobuaki Kikyo

Int. J. Dev. Biol. (2010) 54: 1589-1596

S. macrurus myogenic regulatory factors (MRFs) induce mammalian skeletal muscle differentiation; evidence for functional conservation of MRFs

Hyun-Jung Kim, Robert Güth, Colleen B. Jonsson and Graciela A. Unguez

Int. J. Dev. Biol. (2009) 53: 993-1002

$$
5 \text { yr ISI Impact Factor }(2010)=2.961
$$

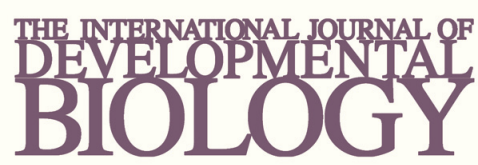

Volume 54 Nos. 6/7

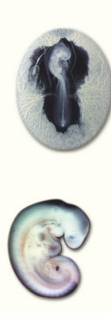

Special Issue

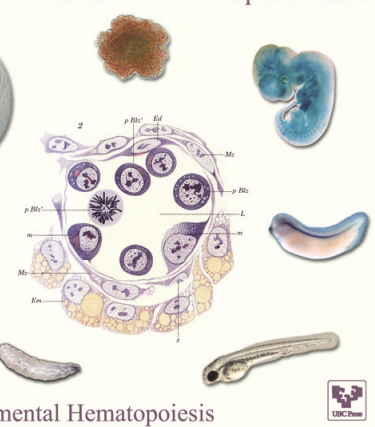

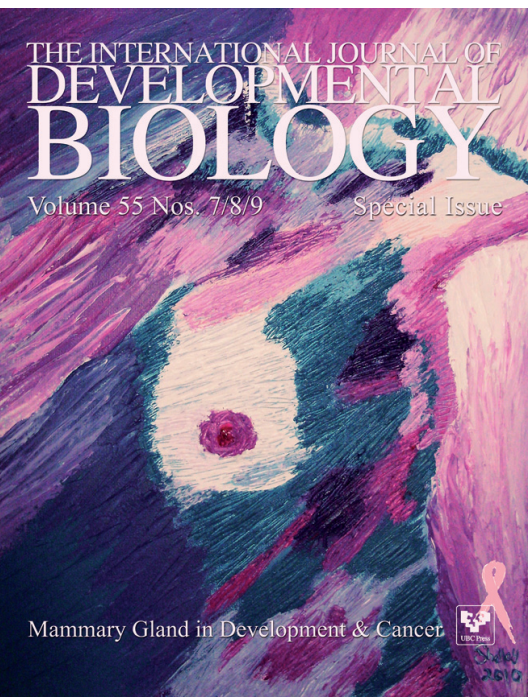

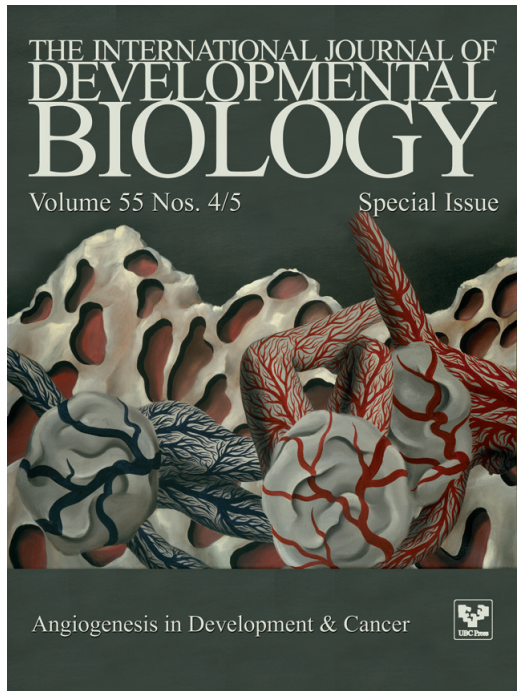

